\title{
Antimatter, Anti-Space, Anti-Time
}

\author{
Alexander Alexandrovich Antonov \\ Independent Researcher, Kiev, Ukraine \\ Email: telan@bk.ru
}

How to cite this paper: Antonov, A.A. (2021) Antimatter, Anti-Space, Anti-Time. Journal of Modern Physics, 12, 646-660. https://doi.org/10.4236/jmp.2021.125042

Received: March 29, 2021

Accepted: April 25, 2021

Published: April 28, 2021

Copyright $\odot 2021$ by author(s) and Scientific Research Publishing Inc. This work is licensed under the Creative Commons Attribution International License (CC BY 4.0).

http://creativecommons.org/licenses/by/4.0/ (c) (i) Open Access

\begin{abstract}
The article shows that the special theory of relativity (STR) created in the last century was based on postulates due to the lack of the required experimental information and turned out to be incorrect, as its principle of light speed non-exceedance was refuted by studies of special processes in linear electric circuits in the 21st century. And thus, it made obsolete the unsuccessful OPERA and ICARUS experiments carried out at the Large Hadron Collider. Therefore, an alternative version of the STR has been proposed. Its relativistic formulas imply the existence of numerous mutually invisible parallel universes and antiverses. It is explained how they can be seen. There is anti-matter, as well as anti-space and anti-time in antiverses in the same quantities as matter, space and time in universes.
\end{abstract}

\section{Keywords}

Imaginary Numbers, Special Theory of Relativity, Invisible Universes, Multiverse, Hyperverse, Antimatter, Anti-Space, Anti-Time

\section{Introduction}

In 1826, when Georg Simon Ohm (1789-1854) discovered the law named after him, the science of physics did not yet exist. There was a natural philosophy. Alexander Grigorievich Stoletov (1839-1896) wrote in this regard: “... physics especially tempted natural philosophers. What a favorable theme were electrical phenomena for the most riotous imaginations ... Attractive and vague deductions were in the foreground: hard work of experimenter and exact mathematical analysis were not honored; they seemed superfluous and harmful in the study of nature ...” And in 1828, Ohm was fired by personal order of Minister of Education for publishing his physics discoveries. The senior official believed that the use of mathematics in physics was unacceptable.

In 1897, Charles Proteus Steinmetz (1865-1923) proposed his interpretation of Ohm's law in respect to linear AC circuits ref. [1]. Now, it is daily used by mil- 
lions of engineers in their practice. Moreover, in addition to its direct purpose of calculating electrical circuits, it also proved physical reality of imaginary numbers in the simplest and most convincing way, and thereby refuted generally accepted version of the special theory of relativity (STR).

However, the STR had to be first created and then refuted. And such a version of the STR was created by efforts of Joseph Larmor (1857-1942) [2], Nobel laureate Hendrik Antoon Lorentz (1853-1928) [3], Jules Henri Poincaré (1854-1912) [4] and Nobel laureate Albert Einstein (1859-1955) [5] in the 20th century. Due to the lack of experimental data required for its creation, that were obtained only in the 21 st century, it was created using the postulates, i.e. assumptions from which the principle of light speed non-exceedance turned out to be incorrect.

But that's not a big deal. Ultimately, all scientific theories are created as a result of identifying and correcting the errors of previously created theories. And then, sooner or later, they are inevitably refuted by subsequent newer theories. Otherwise, science would not have developed. Therefore, this article further proposes a corrected version of the STR.

\section{Refutation of the Principle of Light Speed Non-Exceedance}

Since the principle of light speed non-exceedance in the generally accepted version of the STR, set forth in all university and school textbooks of physics, has still been believed to be true, it will be necessary to explain why this is not so and why this principle, which is just a postulate, since it has never been proven by anyone, turned out to be in demand.

That is because the relativistic formulas obtained in the generally accepted version of the STR couldn't be explained by its creators. For example, the relativistic mass $m$, apparently, takes imaginary values at hyper-light speeds, when $v>c$, in the Lorentz-Einstein formula

$$
m=\frac{m_{0}}{\sqrt{1-\left(\frac{v}{c}\right)^{2}}}
$$

where $m_{0}$ is the rest mass of a moving physical body (e.g. elementary particle);

$m$ is the relativistic mass of a moving physical body;

$v$ is the velocity of a physical body;

$c$ is the speed of light.

However, the authors of the STR did not know how to explain such a result. As well as no one could explain physical meaning of imaginary numbers 400 years before them. Admittedly, today no one can do it so far. Indeed, everyone knows what $2 \mathrm{~kg}$ is, but, no one knows what $2 i \mathrm{~kg}$ is, where $i=\sqrt{-1}$.

Even if the relativistic mass $m$ at hyper-light speeds, when $v>c$, in function (1) corresponded to real numbers, then its graph would still be inexplicable in this velocity range (see Figure 1a), since it corresponds to a physically unstable process that cannot exist in nature. Consequently, formula (1) is incorrect and that is why it could not be explained. 


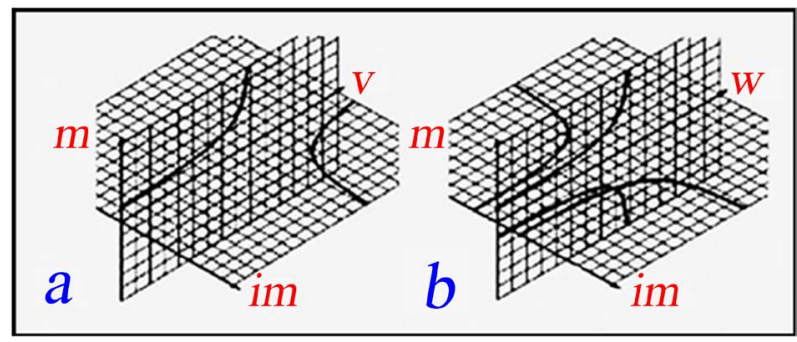

Figure 1. (Color online) Graphs of functions (1) and (2).

After all, physical reality of imaginary numbers has been proven and explained in publications [6]-[21]. In order not to repeat these proofs, we only note that it follows from them that if the principle of light speed non-exceedance were true,

- no shock oscillations such as tsunami, Indian summer, noise of bells, piano music could exist in nature, and even a kid's swing couldn't swing after being pushed by parents;

- there could be no resonance in electric circuits, as well as no electric filters could exist; and thus, there would be neither television, nor telecommunication, nor radiolocation, nor many other things without which modern life would be unthinkable;

- even Ohm's law in Steinmetz's interpretation would not exist.

Since, in accordance with Ohm's law in Steinmetz's interpretation, inductive and capacitive reactances the values of which are imaginary numbers, are measured by the devices available in each radio engineering laboratory, this unambiguously proves their physical reality. After all, it is exactly the ability to register with devices X-ray, radioactive, ultraviolet and infrared radiation, infra and ultrasound, magnetic field, atoms and subatomic particles, as well as many other physical entities that are not registered by the human senses, proves their physical reality. Why, then, a simple and cheap experiment using an ordinary tester in physics is less convincing in solving the problem of proving physical reality of imaginary numbers than the unique expensive OPERA and ICARUS experiments at the Large Hadron Collider?

In fact, since mathematics is the unique universal language of all exact sciences, the correct mathematical interpretation of, let's say, radio engineering and any other experiment is indisputably convincing for all other exact sciences. After all, the Nature is unique, and only people, solely because of their barrenness of intellect, invented many sciences to describe it.

Due to experimental proof of the principle of physical reality of imaginary numbers in the STR, the principle of light speed non-exceedance is no longer required and there is a need for corrected relativistic formulas that allow explaining STR at speeds $v>c$.

\section{Relativistic Formulas of the Corrected Version of the STR}

How can corrected relativistic formulas be obtained? Different approaches can, 
actually, be proposed to solve this issue. And one can reason as follows [22]. The graph of the corrected Lorentz-Einstein function in the range of velocities, must in some respect be similar to the graph of this function in the range of velocities. For example, as in Figure 1b. A simple and understandable analytical description can be offered for such a graph

$$
m=\frac{m_{0}(i)^{q}}{\sqrt{1-\left(\frac{v}{c}-q\right)^{2}}}=\frac{m_{0}(i)^{q}}{\sqrt{1-\left(\frac{w}{c}\right)^{2}}}
$$

where $q=\left\lfloor\frac{v}{c}\right\rfloor$ is the "floor" function of argument $\frac{v}{c}$ in discreet mathematics (see Figure 2a);

$w=v-q c$ is the local velocity (see Figure 2a), the meaning of which will be explained below.

Other relativistic formulas can be corrected in a similar manner.

\section{Explanation of Relativistic Formulas of the Corrected Version of the STR}

A simple explanation can be proposed for the simple formula (2). The quantity $q=0$ obviously corresponds to our visible universe, which is assumed to be the one and the only in the existing version of the STR. However, this version turned out to be incorrect, as its principle of light speed non-exceedance had been refuted.

Therefore, the quantity $q=1$ corresponds to another really existing universe, for which $v=w+c$ follows from $w=v-1 c$, i.e. we get $c \leq v<2 c$ for $0 \leq w<c$. In other words, another adjacent universe is beyond the event horizon and therefore is invisible to us. Therefore, let it for definiteness be called a tachyon universe, like subatomic particles possessing superluminal speed. Herewith, we get $m=m_{0} i$ for a tachyon universe from the formula (2).

By a similar argument let our visible universe be called a tardyon universe. For our tardyon universe $m=m_{0}$.

Subsequently, the quantity $q=2$ corresponds to one more really existing universe, for which $v=w+2 c$ follows from $w=v-2 c$, i.e. we get $2 c \leq v<3 c$ for $0 \leq w<c$. Consequently, this one more universe is also beyond the event horizon and therefore is also invisible to us. It is also invisible to the adjacent universe that is closer to us. Herewith, we get $m=-m_{0}$ for this universe from the formula (2). That is, this universe can be called an antiverse in relation to our universe.

The quantity $q=3$ corresponds to one more really existing universe, for which $v=w+3 c$ follows from $w=v-3 c$, i.e. we get $3 c \leq v<4 c$ for $0 \leq w<c$. Consequently, this universe is also beyond the event horizon and therefore is also invisible to us and to other universes. We get $m=-i m_{0}$ for this universe from the formula (2). And therefore let it be called a tachyon antiverse, etc. 


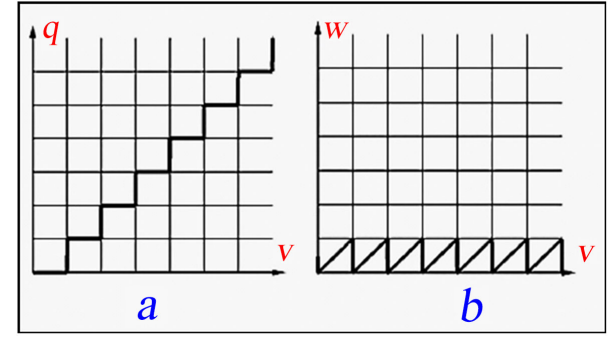

Figure 2. (Color online)Graphs of functions $q(v)$ and $w(v)$.

Hence, it turns out that we live in the Multiverse containing a plenty of mutually invisible universes, rather than in a unique visible universe as asserted in the generally accepted version of the STR. Let this Multiverse be called a hidden Multiverse [23] [24] [25] [26] [27].

\section{Dark Matter, Dark Energy, Dark Space}

Many interesting hypotheses of the Multiverse have been proposed by now [28]-[35]. However, they all are unverifiable, i.e. their truth or falsity can be proven experimentally neither now nor in the distant future. Therefore, they are of limited interest. Another drawback is the fact that they do not anyhow explain extremely incomprehensible phenomena of dark matter and dark energy [36]-[48].

Such extreme incomprehensibility refers also to the hypothesis of the visible Monoverse in the generally accepted version of the STR, about which Albert Einstein spoke very clearly: "Insanity: doing the same thing over and over again and expecting different results"

However, the phenomena of dark matter and dark energy can be quite explicable within the framework of the hypothesis of the hidden Multiverse. Besides the phenomenon of dark space can also be discovered and explained:

- invisibility of dark matter and dark energy is explained by the fact that they are actually neither matter, nor energy, nor any other material physical substance, but only images (though not optical and still less electromagnetic, but gravitational), a sort of a shadow;

- impossibility of detecting any of the chemical elements known to us in the composition of dark matter and dark energy is also explained by the absence of any material content in them, since they are just images;

- at the same time the phenomenon of dark matter is evoked by invisible parallel universes of the hidden Multiverse adjacent to our visible universe;

- the phenomenon of dark energy is evoked by other universes except for our visible universe and invisible parallel universes of the hidden Multiverse adjacent to it;

- in addition, the phenomenon of dark space is similarly evoked by invisible universes outside the hidden Multiverse;

- universes located in and beyond the hidden Multiverse together form the Hyperverse. 


\section{Analysis of WMAP and Planck Spacecraft Data}

Albert Einstein did not exclude such correction of the STR in future. He wrote: "There is no single idea, which I would be sure that it will stand the test of time". And he was absolutely right. After all, if this were not so, then the development of science would be impossible.

An example of the structure of such a hypothetical hidden Multiverse is shown in Figure 3. As can be seen, the universes drifting in the extra spatial dimension are interconnected through portals [49] [50] indicated by single two-sided arrows. The portals arise due to shallow mutual local penetration of the universes into each other. Moreover, the end universes in such a helical structure, evoking the phenomena of dark matter and dark energy, are connected with the universes of dark space.

In order not to repeat the mistake of Albert Einstein due to erroneous assumptions, it is useful to check these results for compliance with the data obtained in the 21st century by the WMAP [51] and Planck [52] spacecraft. According to the WMAP data, the entire universe (in fact, the entire hidden Multiverse, as suggested in the article) is $4.6 \%$ of baryonic matter, $22.4 \%$ of dark matter and $73.0 \%$ of dark energy. According to more recent Planck data, the entire universe (in fact, the entire hidden Multiverse) is $4.9 \%$ of baryonic matter, $26.8 \%$ of dark matter and $68.3 \%$ of dark energy.

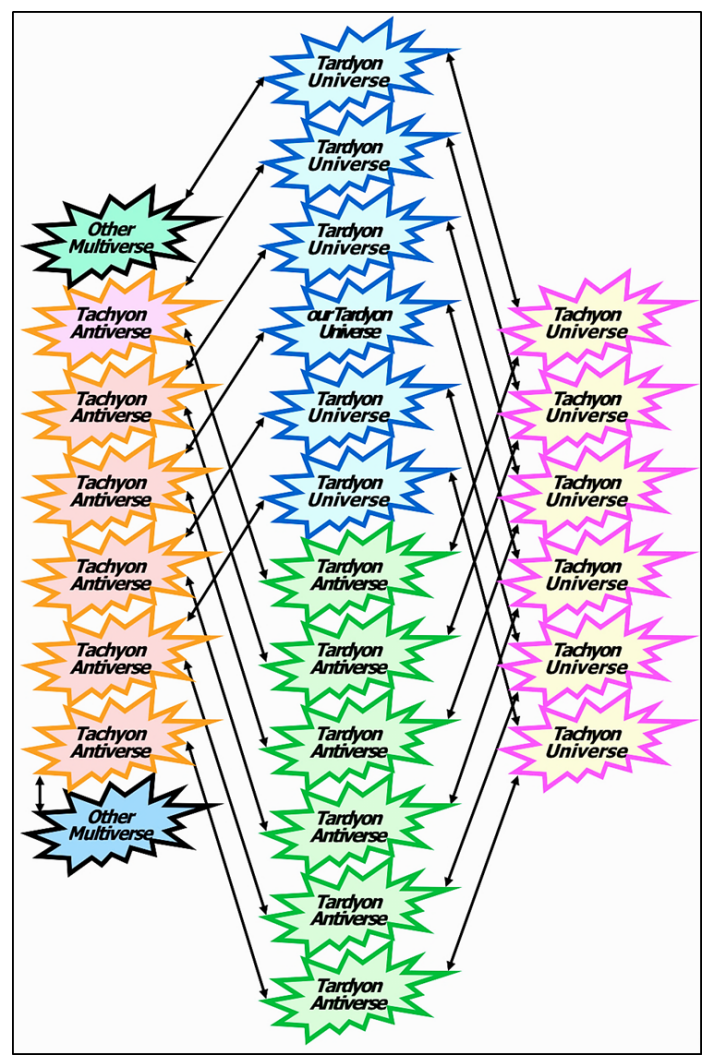

Figure 3. (Color online) The screw structure of the hidden Multiverse corresponding to the formula (2), which illustrates the existence of other Multiverse beyond its borders. 
Based on these data, it is conceivable that mass-energy of parallel universes of the hidden Multiverse has largely averaged over billions of years of existence as a result of the mutual exchange of their micro- and mini-content through the portals (even if for some reason their mass-energy in different universes turned out to be different immediately after the Big Bang) and it is equal to the mass-energy of our visible universe, with precise accuracy.

Thus:

- according to Planck data, the hidden Multiverse contains 100\%/4.9\% = 20.4 parallel universes (according to WMAP data 100\%/4.6\% $=21.8$ parallel universes), i.e. probably 20 ... 22 parallel universes;

- according to Planck data, the hidden Multiverse contains 26.8\%/4.9 = 5.5 parallel universes (according to WMAP data $22.4 \% / 4.6 \%=4.9$ parallel universes), evoking the phenomenon of dark space, i.e. probably 5 .. 6 parallel universes;

- according to Planck data, the hidden Multiverse includes 68.3\%/4.9 = 13.9 parallel universes (according to WMAP data $73.0 \% / 4.6 \%=15.9$ parallel universes), evoking the phenomenon of dark energy, i.e. e. probably14 ... 16 parallel universes.

However, these results do not correspond to the structure of the hidden Multiverse shown in Figure 3, since our visible universe should have not two, but 5 ... 6 adjacent invisible universes.

Admittedly, each tardyon universe in Figure 3 is adjacent to one tachyon universe and one tachyon antiverse. And according to the above mathematical analysis of the data obtained by the WMAP and Planck spacecraft, each tardyon universe should have three tachyon universes and antiverses. Therefore, the assumption that the structure of the hidden Multiverse is described by complex numbers and has one extra spatial dimension turned out to be incorrect. In fact, the hidden Multiverse has three extra dimensions and is described by hyper-complex numbers $f_{q, r, s}(x, y, z)+i_{1} q+i_{2} r+i_{3} s$ [53], where the function $f_{q, r, s}(x, y, z)$ describes distribution of material content of the corresponding parallel universe with coordinates in coordinates $x, y, z$, and the imaginary units $i_{1}, i_{2}, i_{3}$ are connected by the following relations

$$
\begin{gathered}
i_{1}^{2}=i_{2}^{2}=i_{3}^{2}=1 \\
i_{1} i_{2} i_{3}=i_{2} i_{3} i_{1}=i_{3} i_{1} i_{2}=-1 \\
i_{1} i_{3} i_{2}=i_{2} i_{1} i_{3}=i_{3} i_{2} i_{1}=1
\end{gathered}
$$

Lisa Randall wrote in this regard: "We can be living in a three-dimensional space sinkhole in a higher-dimensional universe". And she was right.

\section{Correction of Relativistic Formulas of the Corrected Version of the STR}

Repeatedly corrected relativistic Lorentz-Einstein formula will be written as follows 


$$
m=\frac{m_{0}\left(i_{1}\right)^{q}\left(i_{2}\right)^{r}\left(i_{3}\right)^{s}}{\sqrt{1-\left[\frac{v}{c}-(q+r+s)\right]^{2}}}=\frac{m_{0}\left(i_{1}\right)^{q}\left(i_{2}\right)^{r}\left(i_{3}\right)^{s}}{\sqrt{1-\left(\frac{w}{c}\right)^{2}}}
$$

where $w=v-(q+r+s) c$ is the local velocity for the corresponding universe, which can take values only in the range $0 \leq w \leq c$.

Other relativistic formulas can be corrected in a similar manner [54] [55] [56] [57]

$$
\begin{aligned}
\Delta t & =\Delta t_{0}\left(i_{1}\right)^{q}\left(i_{2}\right)^{r}\left(i_{3}\right)^{s} \sqrt{1-\left[\frac{v}{c}-(q+r+s)\right]^{2}} \\
& =\Delta t_{0}\left(i_{1}\right)^{q}\left(i_{2}\right)^{r}\left(i_{3}\right)^{s} \sqrt{1-\left(\frac{w}{c}\right)^{2}} \\
l & =l_{0}\left(i_{1}\right)^{q}\left(i_{2}\right)^{r}\left(i_{3}\right)^{s} \sqrt{1-\left[\frac{v}{c}-(q+r+s)\right]^{2}} \\
& =l_{0}\left(i_{1}\right)^{q}\left(i_{2}\right)^{r}\left(i_{3}\right)^{s} \sqrt{1-\left(\frac{w}{c}\right)^{2}}
\end{aligned}
$$

The structure of the hidden Multiverse corresponding to the formulas (6), (7), and (8) can be as shown in Figure 4. As can be seen, its quaternionics [58] [59] structure differ from the one shown in Figure 3 in that it contains three tachyon universes $i_{1}, i_{2}, i_{3}$ and three tachyon antiverses $i_{1}, i_{2}, i_{3}$, which provides three required extra dimensions. Thus, the six-dimensional space of the hidden Multiverse (see Figure 5) has three extra dimensions $q, r, s$, where parallel universes are located, and three dimensions $x, y, z$, where material content of each of these universes is located. Moreover, the structure of the hidden Multiverse corresponding to the formulas (6), (7) and (8) differs from the one shown in Figure 3 by the fact that it contains unidirectional portals corresponding to the formulas (4) and (5) in addition to bidirectional portals corresponding to the formula (3).

\section{Antimatter, Anti-Space, Anti-Time}

The 20th century turned out to be rich in outstanding physical discoveries, such as special and general theory of relativity, quantum mechanics, radio electronics, radioactivity, X-ray, dark matter, dark energy, etc. And if radioactivity and X-ray were almost immediately explained and used, dark matter and dark energy have remained unexplained to this day.

Antimatter [42] [60] [61] is another no less incomprehensible astrophysical object than dark matter and dark energy. It is now generally accepted that the Big Bang produced not only matter, but also antimatter. Moreover, they were generated in equal quantities. However, no antimatter has been found in any noticeable quantities in our visible universe. It was obtained only in the form of subatomic antiparticles and some antiatoms, and also was found in some natural phenomena in negligible quantity for a very short time. Synthesis of such anti- 
matter was extremely expensive. Thus, one gram of anti-hydrogen would cost $\$ 662.5$ trillion.

So, where can antimatter in the form of antiverses be found? And does it at all exist anywhere in this form? It cannot apparently be in our visible universe, since otherwise it would annihilate with matter and the universe would be destroyed. By the way, this fact is another refutation of the generally accepted version of the STR. Hence, it can be found only in another universe. And the hidden Multiverse, unlike other hypothetical Multiverse, is quite suitable for this role, since it has antiverses. Moreover, tardyon and tachyon universes and antiverses alternate in the hidden Multiverse in such a way that they assuredly prevent their mutual annihilation. Thus, the hypothesis of the hidden Multiverse completely solves the problem of the existence of antimatter.

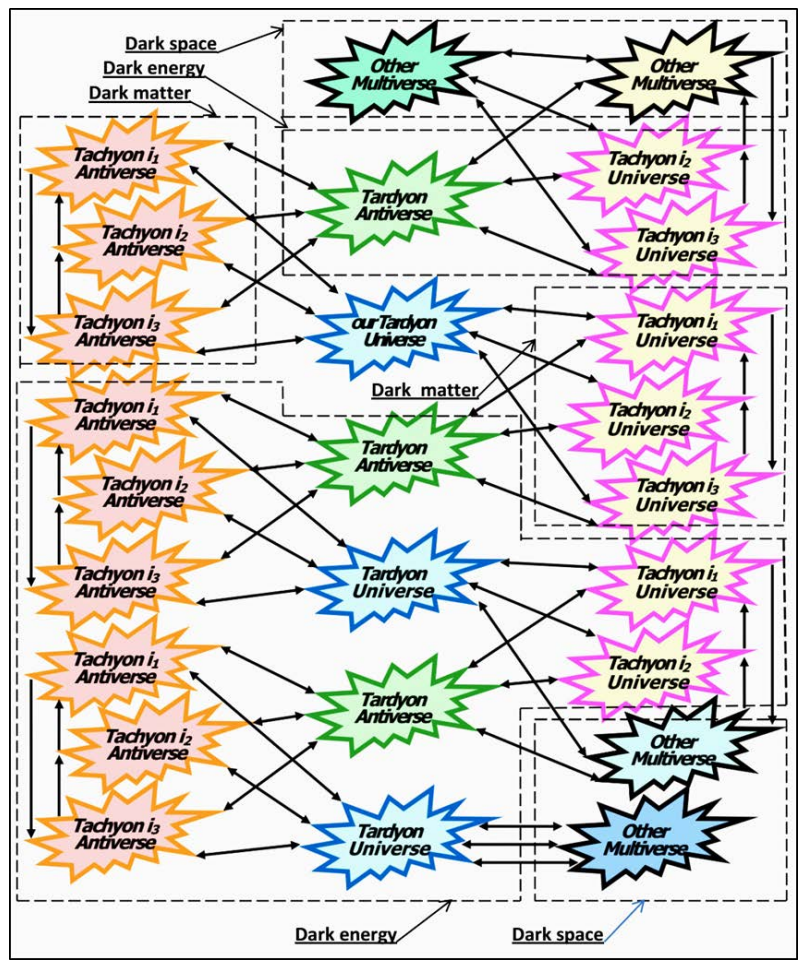

Figure 4. (Color online) The structure of the hidden Multiverse corresponding to the formulas (6), (7) and (8).

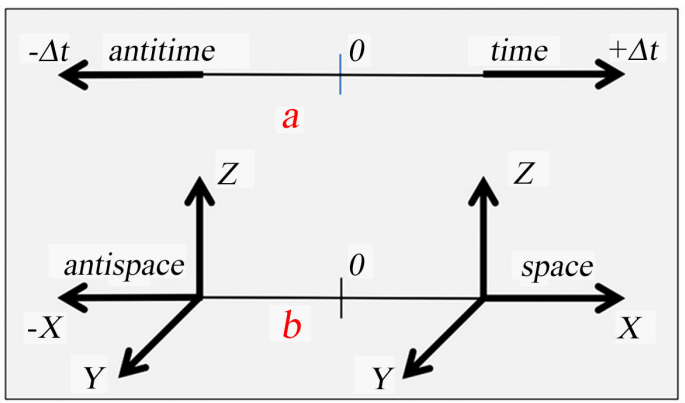

Figure 5. (Color online) Geometric interpretation of the concepts "anti-time" and "anti-space". 
The most interesting thing is that existence of antimatter in antiverses follows from the formula (6), just as existence of anti-space and anti-time in antiverses naturally follows from the formulas (7) and (8). Moreover, people would find nothing unusual in these antiverses, if they got there, since there operate the same physical, chemical and other laws of nature as in our visible universe.

Figure 5 shows a fairly obvious geometric interpretation of these new concepts. As can be seen, time and anti-time differ by the sign of the value appearing in formula (7), and space and anti-space differ by the sign of the value appearing in formula (8). Time and anti-time, in addition, can differ in their different distance on the time axis from the common origin, which depends on the time of occurrence of the corresponding universes and antiverse. Figure 5, for example, depicts a situation in which the universe and the antiverse arose simultaneously.

\section{How to See Invisible Universes}

Thus, the hidden Multiverse is quite unusual in many respects. This arouses some mistrust. Does it exist at all? Nature can give an unequivocal and convincing answer to this question only if its invisible universes are seen. And they can be seen as follows [62] [63].

Since the sky maps of invisible parallel universes are supposedly extremely different, their constellations can be confidently distinguished from those observed in the starry sky by observatories on Earth. Moving along the Earth portals between our visible universe and adjacent invisible universes, one can observe as the star map of one universe is gradually replaced by the star map of the adjacent universe. Therefore, all it takes to make sure of existence of invisible universes is to register differences between the constellations in the starry sky in the portals from the constellations observed in the starry sky outside the portals.

What needs to be done to carry out such an experiment is to find a portal and perform the astronomical observation therein. And although it is clear that entrances to portals are located, at least, in some anomalous zones, which are quite numerous on Earth, no one has yet been engaged in the study of portals directly in portals, since no one has needed it. And besides, it is unsafe, since portals are a sort of invisible labyrinths. Therefore, one can get lost there without an appropriate portal orientation device (similar to marine compass). Such a danger can be minimized, if such observations are carried out at the very entrance to the portal, in the anomalous zone. It is conceivable that some astronomical observatories are already in the anomalous zones, without knowing it. As, for example, the Main Astronomical Observatory of the National Academy of Sciences of Ukraine, which is located in the Holosiivskyi forest, just $12 \mathrm{~km}$ from Kyiv, the capital of Ukraine (see Figure 6).

Therefore, an experiment in detecting invisible universes turns out to be very simple and inexpensive in this case. It consists in comparing computer images of the same area of the starry sky provided by several observatories located close to each other, at least one of which being located in the anomalous zone (see Figure 7); 


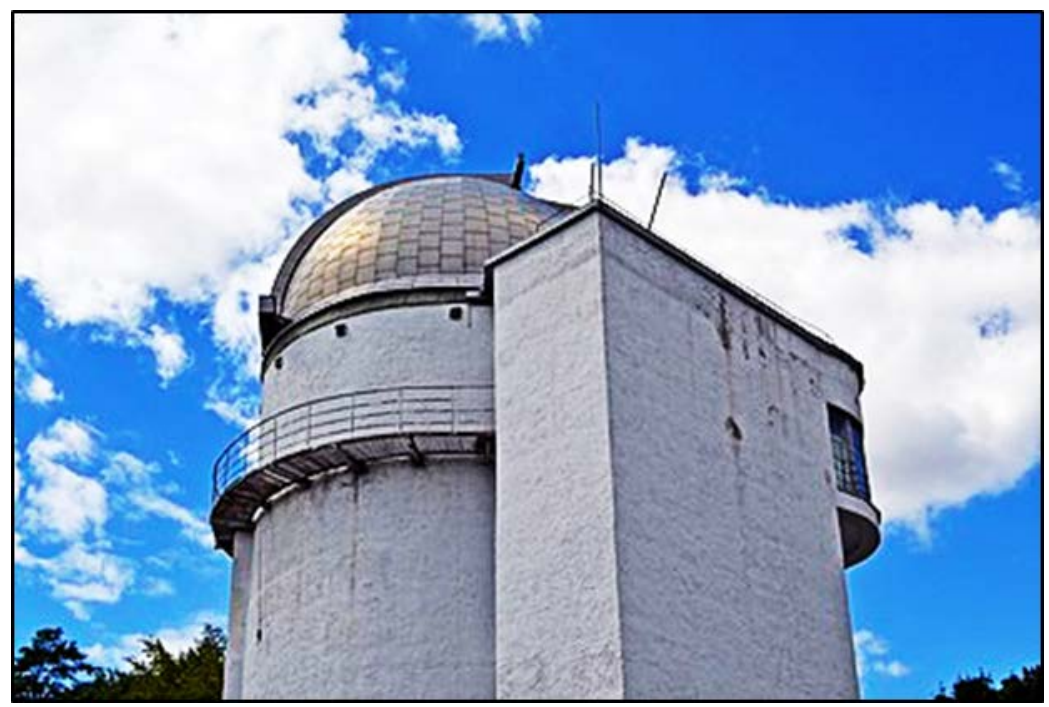

Figure 6. (Color online) Main astronomical observatory of the national academy of science of ukraine.

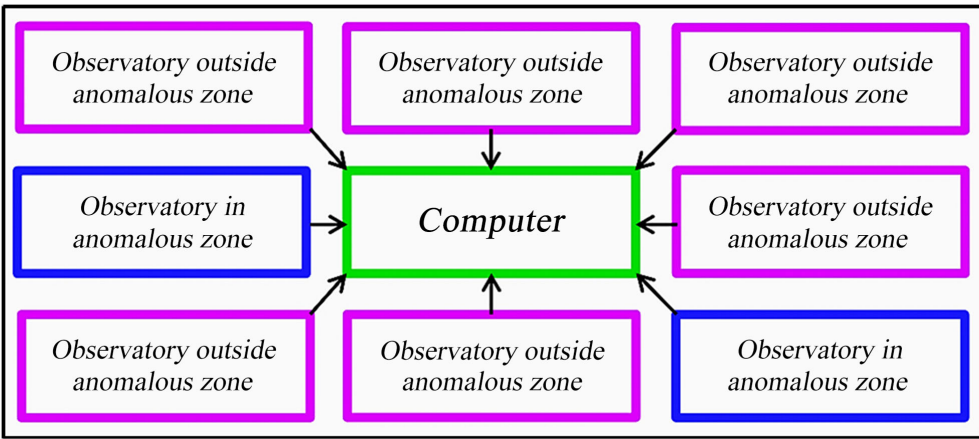

Figure 7. (Color online) Scheme of an experiment to detect invisible universes by identifying differences as a result of comparing the constellations of the starry sky in anomalous zones and outside anomalous zones.

and in revealing differences in the relative position of the stars depicted in these images. If such an experiment is successful, its significance for human civilization will significantly exceed the significance of the discovery of America by Columbus.

\section{Conclusions}

The answer given in the article to one of the questions from the list of unsolved issues of modern physics "where is antimatter?" turned out to be simple and quite logical: it is in the antiverses. At the same time, it has been explained that there are many pairs of universes-antiverses in nature. And therefore, there are many antimatters. Moreover, it has been explained that, in addition to antimatter, there is anti-time and anti-space in the antiverses.

However, the answer to this question has first required the answer to another question from the same list "do invisible parallel universes exist at all?" And the article not only makes it clear that they exist in the Multiverse, which we have 
called hidden, but also why they are parallel and invisible. It also clarifies how and where on Earth invisible universes can be seen.

Notably, the answer to the second question was obtained in the process of answering two more questions from the list of unsolved issues of modern physics "are there extra dimensions?" and "what is dark matter and dark energy?" Due to mathematical analysis of the data obtained by the WMAP and Planck spacecraft, it has been concluded that our hidden Multiverse has a quaternion structure in six-dimensional space. And the phenomenon of dark matter and dark space is explained by the existence in our visible universe of a gravitational wave background generated by the rest of the invisible universes of the hidden Multiverse.

All these answers to the questions from the list of unsolved issues of modern physics became possible after receiving an answer to one more question, although from the list of unsolved issues of modern mathematics, "can imaginary numbers be physically real?" An affirmative answer has been obtained as a result of theoretical and experimental studies of special processes in linear electric circuits, which made it possible to prove the general scientific principle of physical reality of imaginary numbers that, in its turn, refuted the principle of light speed non-exceedance in the STR. And this enabled us to assert that the relativistic formulas obtained in the generally accepted version of the STR are incorrect; they have been incorrectly explained and entailed wrong conclusions. Therefore, attempts to solve the above-mentioned and other physical issues within the framework of this theory were certainly destined for failure.

Thus, it is logical to conclude that the version of the STR presented in textbooks is outdated, since it does not correspond to the experimental data obtained in the 21st century, and therefore it hinders the development of modern physics.

\section{Acknowledgements}

Many famous scientists, such as Albert Einstein, Max Planck, Ernest Rutherford and others, argued that an author does not completely understand his own scientific theory if he is not able to explain it to his wife, mother-in-law and other non-specialists. Therefore, the author of this article, who set himself a goal of presenting the above-presented unconventional approach to solving some issues of astrophysics as clearly as possible, even for non-specialists, used the help of his wife for achieving it. The author expresses appreciation to Olga Ilyinichna Antonova for the help. Being an academic economist, she, nevertheless, took part in discussion of the foregoing alternative version of the STR. Her critical remarks and valuable advice contributed to its more understandable presentation to a general reader.

\section{Conflicts of Interest}

The author declares no conflicts of interest regarding the publication of this paper. 


\section{References}

[1] Steinmetz, C.P. (2010) Theory and Calculation of Electric Circuit. Nabu Press, Charleston.

[2] Larmor, J.J. (1897) Philosophical Transactions of the Royal Society A: Mathematical, Physical and Engineering Sciences, 190, 205-300. https://doi.org/10.1098/rsta.1897.0020

[3] Lorentz, H.A. (1899) Proceedings of the Royal Netherlands Academy of Arts and Sciences, 1, 427-442.

[4] Poincaré, H. (1905) Comptes Rendus, 140, 1504-1508.

[5] Einstein, A. (1905) Annalen der Physik, 17, 891-921. https://doi.org/10.1002/andp.19053221004

[6] Antonov, A.A. (2008) European Journal of Scientific Research, 21, 627-641.

[7] Antonov, A.A. (2009) European Journal of Scientific Research, 28, 193-204.

[8] Antonov, A.A. (2010) General Mathematics Notes, 1, 11-16. https://doi.org/10.17686/sced_rusnauka_2010-887

[9] Antonov, A.A. (2010) International Journal of Pure and Applied Sciences and Technology, 1, 1-12.

[10] Antonov, A.A. (2010) American Journal of Scientific and Industrial Research, 1, 342-349. https://doi.org/10.5251/ajsir.2010.1.2.342.349

[11] Antonov, A.A. (2013) International Journal of Management, IT and Engineering, 3, 219-230. https://doi.org/10.17686/sced_rusnauka_2013-898

[12] Antonov, A.A. (2015) General Mathematics Notes, 31, 34-53. http://www.emis.de/journals/GMN/yahoo_site_admin/assets/docs/4_GMN-9212-V 31N2.1293701.pdf

[13] Antonov, A.A. (2015) Journal of Russian Physical and Chemical Society, 87, 328-355. (In Russian)

[14] Antonov, A.A. (2015) American Journal of Electrical and Electronics Engineering, 3, 124-129.

[15] Antonov, A.A. (2015) Global Journal of Physics, 2, 145-149.

[16] Antonov, A.A. (2016) Ponte Academic Journal, 72, 131-142. https://doi.org/10.21506/j.ponte.2016.7.9

[17] Antonov, A.A. (2016) Journal of Modern Physics, 7, 2299-2313. https://doi.org/10.4236/jmp.2016.716198

[18] Antonov, A.A. (2016) General Mathematics Notes, 35, 40-63.

[19] Antonov, A.A. (2016) International Review of Physics, 10, 31-35.

[20] Antonov, A.A. (2017) Norwegian Journal of Development of the International Science, 6, 50-63.

[21] Antonov, A.A. (2018) Natural Science, 10, 11-30. https://doi.org/10.4236/ns.2018.101002

[22] Antonov, A.A. (2014) American Journal of Scientific and Industrial Research, 5, 40-52.

[23] Antonov, A.A. (2011) British Journal of Science, 2, 51-60. https://doi.org/10.17686/sced_rusnauka_2011-892

[24] Antonov, A.A. (2012) International Journal of Pure and Applied Sciences and Technology, 12, 43-56. https://doi.org/10.17686/sced_rusnauka_2012-896 
[25] Antonov, A.A. (2012) Encyclopedia of Russian Thought. Reports to Russian Physical Society, 16, 3-20. (In Russian)

[26] Antonov, A.A. (2015) International Journal of Advanced Research in Physical Science, 2, 25-32.

[27] Antonov, A.A. (2017) Natural Science, 9, 43-62. https://doi.org/10.4236/ns.2017.93005

[28] Deutch, D. (1998) The Fabric of Reality: The Science of Parallel Universes and Its Implications. Penguin Books, London.

[29] Green, B. (2000) The Elegant Universe: Superstrings, Hidden Dimensions, and the Quest for the Ultimate Theory. Random House Inc., New York. https://doi.org/10.1119/1.19379

[30] Vilenkin, A. (2006) Many Worlds in One: The Search for Other Universes. Hill and Wong, New York.

[31] Steinhardt, P.J. and Turok, N. (2007) Endless Universe: Beyond the Big Bang. Doubleday, New York.

[32] Carr, B. (2009) Universe or Multiverse? Cambridge Univ. Press, Cambridge.

[33] Greene, B. (2011) The Hidden Reality: Parallel Universes and the Deep Laws of the Cosmos. Random House Inc., New York.

[34] Deutsch, D. (2012) The Beginning of Infinity: Explanations That Transform the World. Penguin Books, New York.

[35] Tegmark, M. (2015) Our Mathematical Universe: My Quest for the Ultimate Nature of Reality. Vintage, New York.

[36] Antonov, A.A. (2015) International Journal of Physics, 3, 84-87.

[37] Antonov, A.A. (2015) Global Journal of Science Frontier Research: A Physics and Space Science, 15, 33-38.

[38] Antonov, A.A. (2015) Cosmology, 19, 40-61.

[39] Antonov, A.A. (2015) American Journal of Modern Physics, 4, 1-9. https://doi.org/10.11648/j.ajmp.20150401.11

[40] Antonov, A.A. (2015) American Journal of Modern Physics, 4, 180-188. https://doi.org/10.11648/j.ajmp.20150404.14

[41] Antonov, A.A. (2015) Optics, 4, 43-47.

[42] Antonov, A.A. (2016) Ponte, 72, 288-300. https://doi.org/10.21506/j.ponte.2016.9.22

[43] Antonov, A.A. (2016) Frontiers of Astronomy, Astrophysics and Cosmology, 2, 1-9.

[44] Antonov, A.A. (2016) Journal of Modern Physics, 7, 1228-1246. https://doi.org/10.4236/jmp.2016.710111

[45] Antonov, A.A. (2017) Applied Physics Research, 9, 30-41. https://doi.org/10.5539/apr.v9n2p30

[46] Antonov, A.A. (2017) Journal of Modern Physics, 8, 567-582. https://doi.org/10.4236/jmp.2017.84038

[47] Antonov, A.A. (2018) Journal of Modern Physics, 9, 14-34. https://doi.org/10.4236/jmp.2018.91002

[48] Antonov, A.A. (2019) Journal of Modern Physics, 10, 1006-1028. https://doi.org/10.4236/jmp.2019.108067

[49] Antonov, A.A. (2012) American Journal of Scientific and Industrial Research, 3, 464-473. https://doi.org/10.5251/ajsir.2012.3.6.464.473

[50] Antonov, A.A. (2016) Philosophy and Cosmology, 6, 11-27. (In Russian) 
http://ispcjournal.org/journals/2016-16/Antonov_16.pdf

[51] Hinshaw, G., Larson, D., Komatsu, E., et al. (2013) Nine Year Wilkinson Anisotropy Probe (WMAP) Observations: Cosmological Parameter Results.

[52] Adam, R., Ade, P.A.R., Aghanim, N., et al. (2015) Plank 2015 Results. 1. Overview of Products and Scientific Results.

[53] Kantor, I.L. and Solodovnikov, A.S. (1989) Hypercomplex Numbers. Springer Verlag, Berlin. https://doi.org/10.1007/978-1-4612-3650-4

[54] Antonov, A.A. (2020) Journal of Russian Physicochemical Society, 91, 57-94. (In Russian)

[55] Antonov, A.A. (2020) Journal of Modern Physics, 11, 324-342. https://doi.org/10.4236/jmp.2020.112020

[56] Antonov, A.A. (2020) Journal of Russian Physicochemical Society, 92, 39-72. (In Russian)

[57] Antonov, A.A. (2021) German International Journal of Modern Science, 4, 38-47.

[58] Antonov, A.A. (2015) Global Journal of Science Frontier Research: A Physics and Space Science, 15, 8-15.

[59] Antonov, A.A. (2020) Österreichisches Multiscience Journal (Innsbruck, Austria), 35, 61-72. http://osterr-science.com

[60] Alfvén, H. (1966) Worlds-Antiworlds: Antimatter in Cosmology. W. H. Freeman \& Co., San Francisco.

[61] Frazer, G. (2004) Antimatter: The Ultimate Mirror. Cambridge University Press, Cambridge.

[62] Antonov, A.A. (2020) Journal of Modern Physics, 11, 593-607. https://doi.org/10.4236/jmp.2020.115039

[63] Antonov, A.A. (2020) Natural Science, 12, 569-587. https://doi.org/10.4236/ns.2020.128044 